\title{
Calculations of Energy Levels Using the Weakest Bound Electron Potential Model Theory
}

\author{
T.-Y. ZHANG AND N.-W. ZHENG* \\ Department of Chemistry, University of Science and Technology of China
}

Hefei, Anhui 230026, P.R. China

(Received November 29, 2008)

\begin{abstract}
In this paper, we introduce a new method for calculation of energy levels in detail and give our results for several iso-spectrum-level series as examples: [He] $2 s 2 p{ }^{1} P_{1}$, [He] $2 s 2 p{ }^{3} P_{0}$, [He] $2 s 2 p{ }^{3} P_{2}$, and [He] $2 s 3 s{ }^{3} S_{1}$ series of Be-like sequence; [Ne] $3 s^{2} 3 d^{2} D_{3 / 2}$ series and [Ne] $3 s^{2} 3 d^{2} D_{5 / 2}$ series of Al-like sequences; [Ne] $4 p{ }^{2} P_{1 / 2}$ series, [Ne] $5 d^{2} D_{5 / 2}$ series, and $[\mathrm{Ne}] 6 f^{2} F_{7 / 2}$ series of Na-like sequences. In the method $I(Z)=T_{\lim }(Z)-T(Z, n)$, where $I(Z), T_{\lim }(Z)$, and $T(Z, n)$ denote ionization potential, series limit, and energy level of a given member, respectively. The expression of non-relativistic part of $I(Z)$ is derived from weakest bound election potential model theory and relativistic effects of $I(Z)$ are included by using a six-order polynomial in $Z$. Our results are compared with the experimental data and with those obtained by other theoretical method.
\end{abstract}

PACS numbers: 01.55.+b, 31.15.-p

\section{Introduction}

The energy levels are widely applied in many fields, such as plasma diagnosis, astrophysics, laser development, and analytical chemistry. Therefore, more and more attention has been paid on calculations of high-precision energy levels of atoms and ions. Many theoretical methods have been developed in this field: multiconfiguration Hartree-Fock (MCHF) method [1-3], multiconfiguration Dirac-Hartree-Fock (MCDHF) method [1-3], configuration interaction (CI) method [4-7], relativistic many-body perturbation theory (RMBPT) [8-14], and weakest bound election potential model (WBEPM) theory [15-23], etc.

In recent years, many excellent works have been done by theoretical researchers. The MCHF method has been widely used to calculate properties of atoms and ions including energy levels. Irimia and Fischer reported energy levels and transition probabilities of neutral argon using both MCHF method and MCDHF method [1]. Fischer and Tachiev also applied MCHF method to the study of energy levels and lifetimes for the Be-like to Ne-like sequences [2]. Two years later, the same group reported an extension of this work to the sequences with 11-18 electrons [3]. In their calculations, the MCHF approach was used for obtaining the "best" radial functions for the interacting terms and the relativistic effects were included through the Breit-Pauli approximation. Dzuba et al. used both Hartree-Fock (HF) and CI method to study the energy levels and lifetimes of $\mathrm{Nd}(\mathrm{IV}), \mathrm{Pm}(\mathrm{IV})$, $\mathrm{Sm}(\mathrm{IV})$, and $\mathrm{Eu}(\mathrm{IV})$ [4]. They also reported energy levels of $\mathrm{Ge}, \mathrm{Sn}, \mathrm{Pb}[5]$ and barium and radium $[6,7]$. They started their calculations from the relativistic Hatree-

* corresponding author; e-mail: nwzheng@ustc.edu.cn
Fock method and used CI method to treat the interaction between valence electrons. Another powerful method in this field is RMBPT. Yb-, Al-, Ga-like ions have been investigated by this method [8-10]. Energy levels of silver, beryllium, magnesium, francium and zinc isoelectronic sequences were also studied by this method [11-14]. The core-valence correlations are included beyond the secondor third-order of RMBPT. Since the WBEPM theory was proposed [15], many studies have been performed to calculate energy levels for atoms and ions [16-24]. In these works, the authors used the concept of the spectrum-level-like series and a new formula taking perturbation into account for calculation. Excellent results are obtained in those works. Deviations between the theoretical results and experimental values are generally smaller than $1 \mathrm{~cm}^{-1}$.

The purpose of this paper is to introduce another new method to calculate energy levels proposed by one of authors (N.W.Z), recently [25]. After introducing the concept of iso-spectrum-level series, we give the results of several iso-spectrum-level series including $\mathrm{Be}-, \mathrm{Al}-$, and Na-like sequence as examples. According to the WBEPM theory, the expression of nonrelativistic ionization potential is written as a function with two-order in $Z$. The relativistic effects are included by using a six-order polynomial in $Z$. We compare our results with the experimental data and with those obtained by other theoretical method.

\section{The conception of the iso-spectrum-level series}

In our previous works, the conception of the iso-spectrum-level series has already been proposed and used to calculate the ionization potential [26-29]. In order to describe our method clearly, we introduce the conception of the iso-spectrum-level series first. The conception of iso-electronic series is usually used in the study 
for the ionization energies of ground state. All members (atom and ions) in an isoelectronic series have the same electron configuration. As a result, the conception of isoelectronic series is associated with the electron configuration and cannot provide the information relating to the terms or energy levels. However, for the study of ground state, all systems lie in the lowest energy state. Therefore, the conception of isoelectronic series is convenient to investigate the regularities of ionization energies of ground state. But for the atoms or ions with excited electrons, each electron configuration usually gives rise to several terms and each term splits into several spectral levels further. Under this situation, the conception of iso-electronic series is too rough to study the energies. Therefore, we introduce the conception of the iso-spectrum-level series study of the general regulations of excited states of atoms. An iso-spectrum-level series is a series of energy levels that is composed of energy levels with the same spectral level symbol in a given iso-electronic series. For example, $\mathrm{Be}(\mathrm{I})\left([\mathrm{He}] 2 s 3 p{ }^{3} P_{1}\right)$, $\mathrm{B}(\mathrm{II})\left([\mathrm{He}] 2 s 3 p{ }^{3} P_{1}\right), \ldots, \mathrm{O}(\mathrm{V})\left([\mathrm{He}] 2 s 3 p{ }^{3} P_{1}\right), \ldots$, make up an iso-spectrum-level series named $\mathrm{Be}(\mathrm{I})([\mathrm{He}]$ $\left.2 s 3 p{ }^{3} P_{1}\right)$. From the definition, in a given iso-spectrum-level series, not only the electron configuration but also the spectrum energy levels is defined. The only variable parameter is the nuclear charge $Z$. Therefore, the ionization energy of WBE in a given iso-spectrum-level series could be approximate to a function of nuclear charge $Z$.

\section{Theory and method}

WBEPM theory is based on the consideration of the dynamic successive ionization, the choice of zero energy in quantum mechanics and the separation of the weakest bound electron (WBE) and non-weakest bound electrons (NWBE) $[15,16]$.

A free particle with $N$ electrons and a nucleus of charge $+Z e$ in its ground state can give rise to $N$ stages of ionization. The ionized species in $N$ successive ionization stages are, respectively, neutral atom, unipositive ion, $\ldots,+(Z-1)$ ion. The conception of WBE is referred to the definition of the ionization of a free particle: the ionization potential for a free particle is defined as the energy required completely to move the WBE from the particle. Therefore, we classified the electrons in an atom or ion system into two types, WBE and NWBE. The WBE is the electron which is most weakly bound to the system and excited or ionized first, the rest electrons are called NWBE. In terms of excitation or ionization, the WBE in a given system differs from the rest of the electrons in behavior. We can separate the WBE and the NWBE, and the problem of the WBE can be treated as a one-electron problem. Each electron in the $N$-electrons system acts sooner or later as a WBE in the ionization procedure. By removing the first, the second, .. N-th WBE, an $N$-electrons atom can give $N$ stages of ionization. Each stage of successive ionization processes corresponds to the removal of a WBE from the related subsystem. Accurate treatment of the WBE can provide accurate knowledge of atomic and ionic properties.

As the ionization energy is defined as the energy required completely removing the weakest bound electron from an atom or an ion in its ground or excited states, the energy of a level in spectrum-level-like series [17-24] can be written as

$$
T(n)=T_{\lim }-I_{\mathrm{exp}},
$$

where $T_{\lim }$ is the ionization limit for a spectrum-level-like series. $I_{\text {exp }}$ is the ionization energy of WBE. In order to get the value of $T(n)$ the value of $I_{\exp }$ is needed. In this work, we use the theoretical value of ionization energy, $I_{\text {cal }}$, to replace the $I_{\text {exp }}$. Therefore, we get

$$
T(n)=T_{\text {lim }}-I_{\text {cal }} \text {. }
$$

We divided approximately the ionization energy into nonrelativistic part and relativistic part [30]

$$
I_{\text {cal }}=I_{\mathrm{nr}}+I_{\mathrm{r}} \text {, }
$$

where $I_{\mathrm{nr}}$ represents the nonrelativistic energy and $I_{\mathrm{r}}$ represents relativistic energy.

Now we employ the WBEPM theory to calculate the nonrelativistic energy $I_{\mathrm{nr}}$.

According to WBEPM theory $[15,16]$, the Schrödinger equation of $\mathrm{WBE}$ is

$$
\left[-\frac{1}{2} \nabla_{i}^{2}+V\left(r_{i}\right)\right] \varphi_{i}=\varepsilon_{i} \varphi_{i} .
$$

In WBEPM theory the potential function $V\left(r_{i}\right)$ in Eq. (4) may be written as (in atomic units)

$$
V\left(r_{i}\right)=\frac{-Z^{\prime}}{r_{i}}+\frac{d(d+1)+2 d l}{2 r_{i}^{2}},
$$

where $Z^{\prime}$ is the effective nuclear charge, $l$ is the angular quantum number of $\mathrm{WBE}$, and $r_{i}$ is the distance between the WBE $i$ and the nucleus. Parameter $d$ is introduced to modify the integral quantum number $n_{i}$ and angular quantum number $l_{i}$ into nonintegral $n_{i}^{\prime}$ and $l_{i}^{\prime}$.

Substituting Eq. (5) into Eq. (4) and solving the Schrödinger equation of the WBE, we can obtain the following expressions of energy eigenvalue and the radial function:

$$
\varepsilon_{i}=-\frac{Z_{i}^{\prime 2}}{2 n_{i}^{\prime 2}}
$$

and

$$
R=C \exp \left(-\frac{Z^{\prime} r}{n^{\prime}}\right) r^{l^{\prime}} L_{n-l-1}^{2 l^{\prime}+1}\left(\frac{2 Z^{\prime} r}{n^{\prime}}\right),
$$

where $n^{\prime}$ is the effective principal quantum number with $n^{\prime}=n+d, l^{\prime}$ is the effective angular quantum number with $l^{\prime}=l+d, C$ is the normalization factor, and $L_{n-l-1}^{2 l^{\prime}+1}\left(\frac{2 Z^{\prime} r}{n^{\prime}}\right)$ is the generalized Laguerre polynomial.

Because Eq. (4) is the non-relativistic one-electron Schrödinger equation, the energy eigenvalue of WBE obtained from Eq. (4) negative value of the non-relativistic part of ionization energy, $I_{\mathrm{nr}}$ :

$$
I_{\mathrm{nr}}=-\varepsilon_{i}=\frac{Z_{i}^{\prime 2}}{2 n_{i}^{\prime 2}} .
$$

For an iso-spectrum-level series we can write Eq. (1) 
into

$$
T(Z, n)=T_{\lim }(Z)-I_{\exp }(Z),
$$

and the effective nuclear charge $Z^{\prime}$ was proposed as a function concerning nuclear charge $Z$ [15], that is

$$
Z^{\prime}=\sqrt{(Z-\sigma)^{2}+g\left(Z-Z_{0}\right)},
$$

where $Z_{0}$ is the nuclear charge of the first member in an iso-spectrum-level series, for example, $Z_{0}$ is the nuclear charge of the Be atom for iso-spectrum-level series $\mathrm{Be}(\mathrm{I})$ ([He] $\left.2 s 3 p^{3} P_{1}\right), \sigma$ is the screening constant of the first member, and relatively increase factor $g$ is a parameter that indicates the effect on the effective nuclear charge due to the increase in the nuclear charge in series.

The non-relativistic ionization energy can be written as

$$
I_{\mathrm{nr}}(Z)=\frac{(Z-\sigma)^{2}+g\left(Z-Z_{0}\right)}{2 n^{\prime 2}} .
$$

In order to obtain the values of parameters $n^{\prime}, \sigma$, and $g$, we considered the first difference of the non-relativistic ionization potential. In an iso-spectrum-level series, the plot of the first difference of the ionization potential, $\Delta I_{\mathrm{nr}}=I_{\mathrm{nr}}(Z+1)-I_{\mathrm{nr}}(Z)$, vs. nuclear charge $Z$, would be a straight line. Because the relativistic part of ionization potential is quite small, the plot of the first differences of the experimental ionization potential $\Delta I_{\exp }=I_{\exp }(Z+1)-I_{\exp }(Z)$ vs. nuclear charge $Z$ could be approximated to the plot of the first difference of the ionization potential, $\Delta I_{\mathrm{nr}}=I_{\mathrm{nr}}(Z+1)-I_{\mathrm{nr}}(Z)$, vs. nuclear charge $Z$. In this work, the experimental data are taken from Ref. [31]. Therefore, the effective principle quantum number $n^{\prime}$ can be treated as a constant approximately, and can be obtained from the plot of $\Delta I_{\exp }$ and nuclear charge $Z$. The screening constant of the first member $\sigma$ and the relatively increase factor $g$ can be calculated later. Therefore, the energy levels can be obtained from the following equation:

$$
T(Z, n) \approx T_{\lim }(Z)-\frac{(Z-\sigma)^{2}+g\left(Z-Z_{0}\right)}{2 n^{\prime 2}} .
$$

As $Z^{2}$ is the highest power of Eq. (12), the relativistic effects such as mass velocity, the Darwin term, and the spin-orbit term cannot be included completely in Eq. (12). In order to get more accurate energies, the relativistic effects must be taken into account. As mentioned above, in a given iso-spectrum-level series, the only variable parameter is the nuclear charge $Z$. Therefore, in this work, the relativistic energies are presented as a univariant function of a six-order polynomial in nuclear charge

$$
I_{\mathrm{r}}(Z)=\sum_{0}^{6} a_{i} Z^{i}
$$

We considered the deviations between experimental ionization potential $I_{\exp }(Z)$ and the ionization potential $I_{\mathrm{nr}}(Z)$ calculated from Eq. (11) are equal to the relativistic part $I_{\mathrm{r}}(Z)$. Here experimental values of ionization potential are taken from NIST data base [31]. By fitting deviations $I_{\exp }(Z)-I_{\mathrm{nr}}(Z)$ to Eq. (13), we can obtain the values of coefficients $a_{i}(i=0-6)$. The theoretical value of ionization energy can be expressed as the following equation:

$$
I_{\text {cal }}(Z)=\frac{(Z-\sigma)^{2}+g\left(Z-Z_{0}\right)}{2 n^{\prime 2}}+\sum_{0}^{6} a_{i} Z^{i} .
$$

Then for an iso-spectrum-level series, we obtain

$$
\begin{aligned}
& T(n)=T_{\lim } \\
& -\left[\frac{(Z-\sigma)^{2}+g\left(Z-Z_{0}\right)}{2 n^{\prime 2}}+\sum_{0}^{6} a_{i} Z^{i}\right] .
\end{aligned}
$$

As the $Z$ is determined for a given member, Eq. (14) reduces as Eq. (2) and one can calculate $T(n)$ through $I_{\text {cal }}$ and $T_{\text {lim }}$.

\section{Results and discussion}

We studied the energy levels of iso-spectrum-level series along Be-, Al-, and Na-like sequences using the method mentioned above. The parameters needed in Eq. (15) are listed in Tables I-II, and the results of this work are listed in Tables III-IX.

\section{TABLE I}

Parameters of Eq. (14) for [He] $2 s 2 p{ }^{1} P_{1}$; [He] $2 s 2 p{ }^{3} P_{0}$; [He] $2 s 2 p{ }^{3} P_{2}$; and $[\mathrm{He}] 2 s 3 s{ }^{3} S_{1}$ series of Be-like sequences.

\begin{tabular}{c|c|c|c|c}
\hline \hline Parameters & {$[\mathrm{He}] 2 s 2 p{ }^{1} P_{1}$} & {$[\mathrm{He}] 2 s 2 p{ }^{3} P_{0}$} & {$[\mathrm{He}] 2 s 2 p{ }^{3} P_{2}$} & {$[\mathrm{He}] 2 s 3 s{ }^{3} S_{1}$} \\
\hline$\sigma$ & 2.91379 & 2.61381 & 2.60991 & 2.62907 \\
$g$ & 0.397358 & 0.295579 & 0.32755 & 0.338591 \\
$n^{\prime}$ & 1.99205 & 1.9906 & 1.99627 & 2.98728 \\
$a_{0}$ & 73035.1 & 22114.3 & 24315.3 & 11693.9 \\
$a_{1}$ & -43659.7 & -14188 & -14082 & -7217.97 \\
$a_{2}$ & 9724.99 & 3392.89 & 2996.71 & 1634.36 \\
$a_{3}$ & -1062.34 & -388.985 & -310.527 & -173.436 \\
$a_{4}$ & 61.5715 & 22.9618 & 17.0618 & 9.2005 \\
$a_{5}$ & -1.81346 & -0.679121 & -0.475133 & -0.236533 \\
$a_{6}$ & 0.0212893 & 0.00817741 & 0.00520787 & 0.00243893
\end{tabular}


TABLE II

Parameters of Eq. (14) for [Ne] $3 s^{2} 3 d^{2} D_{3 / 2}$ series, [Ne] $3 s^{2} 3 d^{2} D_{5 / 2}$ series, and [Ne] $3 s^{2} 3 d^{2} D_{5 / 2}$ series of Al-like sequences and [Ne] $4 p^{2} P_{1 / 2}$ series, [Ne] $5 d^{2} D_{5 / 2}$ series, and $[\mathrm{Ne}] 6 f{ }^{2} F_{7 / 2}$ series of Na-like sequences.

\begin{tabular}{c|c|c|c|c|c}
\hline \hline Parameters & \multicolumn{3}{|c|}{ Al-like sequences } & \multicolumn{2}{c}{ Na-like sequences } \\
\hline & {$[\mathrm{Ne}] 3 s^{2} 3 d^{2} D_{3 / 2}$} & {$[\mathrm{Ne}] 3 s^{2} 3 d^{2} D_{5 / 2}$} & {$[\mathrm{Ne}] 4 p^{2} P_{1 / 2}$} & {$[\mathrm{Ne}] 5 d^{2} D_{5 / 2}$} & {$[\mathrm{Ne}] 6 f^{2} F_{7 / 2}$} \\
\hline$\sigma$ & 11.9176 & 11.9172 & 9.76373 & 10.0198 & 10.0016 \\
$g$ & 0.0958325 & 0.0994782 & 0.640162 & -0.0152469 & -0.00462789 \\
$n^{\prime}$ & 2.84873 & 2.84992 & 3.87277 & 4.88813 & 5.98929 \\
$a_{0}$ & 20494300 & 17658700 & 646086 & -93157 & 1920.37 \\
$a_{1}$ & 6179120 & 5245450 & 199379 & 40195.8 & -508.401 \\
$a_{2}$ & 764622 & 638337 & 24607.5 & -6764.28 & 38.3766 \\
$a_{3}$ & 49786.7 & 40807.9 & 1561.9 & 570.524 & 0.785963 \\
$a_{4}$ & 1801.3 & 1447.43 & 54.3212 & -25.5031 & -0.240012 \\
$a_{5}$ & 34.3498 & 27.0199 & 0.994358 & 0.575745 & 0.0110767 \\
$a_{6}$ & 0.269627 & 0.207276 & 0.00749563 & -0.00520289 & -0.000161941
\end{tabular}

TABLE III

Energy levels $\left[\mathrm{cm}^{-1}\right]$ for the $[\mathrm{He}] 2 s 2 p{ }^{1} P_{1}$ series of Be-like sequences.

\begin{tabular}{c|c|c|c}
\hline \hline$Z$ & $\begin{array}{c}\text { Experimental } \\
\text { results [31] }\end{array}$ & $\begin{array}{c}\text { Present } \\
\text { results }\end{array}$ & $\begin{array}{c}\text { MBPT } \\
\text { method [32] }\end{array}$ \\
\hline 4 & 42565.4 & 42566.8 & 38316 \\
5 & 73396.5 & 73326.2 & 70046 \\
6 & 102352 & 102472 & 99710 \\
7 & 130694 & 130746 & 128534 \\
8 & 158798 & 158722 & 156979 \\
9 & 186844 & 186739 & 185279 \\
10 & 214952 & 214950 & 213581 \\
11 & 243208 & 243541 & 241993 \\
12 & 271687 & 270861 & 270607 \\
13 & 300490 & 300947 & 299511 \\
14 & 329679 & 330319 & 328790 \\
15 & 359343 & 359247 & 358537 \\
16 & 389583 & 388965 & 388827 \\
17 & 420501 & 419469 & 419799 \\
18 & 452212 & 450993 & 451567 \\
19 & 484800 & 486375 & 484250 \\
20 & 518620 & 519007 & 517980 \\
21 & 553440 & 546768 & 552911 \\
22 & 589692 & 589446 & 589206 \\
23 & 627500 & 626535 & 627054 \\
24 & 667080 & 667130 & 666658 \\
& & &
\end{tabular}

TABLE IV Energy levels $\left[\mathrm{cm}^{-1}\right]$ for the $[\mathrm{He}] 2 s 2 p{ }^{3} P_{0}$ series of Be-like sequences.

\begin{tabular}{c|c|c|c}
\hline \hline$Z$ & $\begin{array}{c}\text { Experimental } \\
\text { results [31] }\end{array}$ & $\begin{array}{c}\text { Present } \\
\text { results }\end{array}$ & $\begin{array}{c}\text { MBPT } \\
\text { method [32] }\end{array}$ \\
\hline 4 & 21978.3 & 22008.5 & 20610 \\
5 & 37335.5 & 37285.2 & 36624 \\
6 & 52367.1 & 52354.3 & 51916 \\
7 & 67209.2 & 67206.9 & 66898 \\
8 & 81942.5 & 81946.8 & 81718 \\
9 & 96590 & 96649.6 & 96447 \\
10 & 111253 & 111347 & 111128 \\
11 & 125880 & 126189 & 125785 \\
12 & 140504 & 139525 & 140437 \\
13 & 155148 & 155399 & 155095 \\
14 & 169802 & 170310 & 169765 \\
15 & 184478 & 184461 & 184456 \\
16 & 199181 & 198973 & 199145 \\
17 & 213913 & 213671 & 213877 \\
18 & 228674 & 228580 & 228649 \\
19 & 243520 & 246307 & 243460 \\
20 & 258290 & 260020 & 258304 \\
21 & 273200 & 267420 & 273185 \\
22 & 288190 & 288209 & 288103 \\
23 & 303100 & 301950 & 303062 \\
24 & 318030 & 318023 & 318076 \\
& & &
\end{tabular}


TABLE V

Energy levels $\left[\mathrm{cm}^{-1}\right]$ for the $[\mathrm{He}] 2 s 2 p{ }^{3} P_{2}$ series of Be-like sequences.

\begin{tabular}{c|c|c|c}
\hline \hline$Z$ & $\begin{array}{c}\text { Experimental } \\
\text { results [31] }\end{array}$ & $\begin{array}{c}\text { Present } \\
\text { results }\end{array}$ & $\begin{array}{c}\text { MBPT } \\
\text { method [32] }\end{array}$ \\
\hline 4 & 21981.3 & 22017.5 & 20614 \\
5 & 37357.8 & 37293.4 & 36648 \\
6 & 52447.1 & 52433.7 & 51997 \\
7 & 67416.3 & 67424.9 & 67107 \\
8 & 82385.3 & 82400.1 & 82164 \\
9 & 97427 & 97486.8 & 97287 \\
10 & 112702 & 112784 & 112578 \\
11 & 128218 & 128514 & 128128 \\
12 & 144091 & 143108 & 144030 \\
13 & 160429 & 160691 & 160381 \\
14 & 177318 & 177842 & 177281 \\
15 & 194856 & 194855 & 194841 \\
16 & 213182 & 212945 & 213157 \\
17 & 232413 & 232050 & 232389 \\
18 & 252683 & 252330 & 252668 \\
19 & 274090 & 276562 & 274133 \\
20 & 296950 & 298132 & 296932 \\
21 & 321240 & 315023 & 321230 \\
22 & 347260 & 347297 & 347198 \\
23 & 375000 & 374979 & 375029 \\
24 & 405020 & 408026 & 404922 \\
& & &
\end{tabular}

Table I presents the parameters for the Be-like series including [He] $2 s 2 p{ }^{1} P_{1}$; [He] $2 s 2 p{ }^{3} P_{0}$; [He] $2 s 2 p{ }^{3} P_{2}$; and $[\mathrm{He}] 2 s 3 s{ }^{3} S_{1}$. For these series, the experimental data from $Z=4$ to $Z=16$ are used to obtain the parameters $n^{\prime}, \sigma$, and $g$. The experimental data are taken from Ref. [31]. The effective principle quantum number $n^{\prime}$ is obtained from the first differences of $I_{\exp }(Z) . \sigma$ is the screening constant of the first member of a given iso-spectrum-level series, and we calculated it from Eq. (12). $g$ is called relative increase factor which indicates the effect on the effective nuclear charge. Each member in a iso-spectrum-level series is used to obtain the relative $g_{i}$, and $g$ is the arithmetical average of the $g_{i}$. When obtained the parameters $a_{i}(i=0-6)$ from the least-squares fitting, $Z=20,22,24$ are added for $[\mathrm{He}] 2 s 2 p$ ${ }^{1} P_{1}$ series; $Z=22,24$ are added for $[\mathrm{He}] 2 s 2 p{ }^{3} P_{0}$ series; $Z=22,23$ are added for $[\mathrm{He}] 2 s 2 p{ }^{3} P_{2}$ series; and $Z=22,24$ are added for [He] $2 s 3 s{ }^{3} S_{1}$ series. Results of these Be-like series are listed in Tables III-VI. All the energies are given in $\mathrm{cm}^{-1}$ unit. We compared our results with the experimental data and those obtained by Safronova et al. [32, 33]. In each table, column 1 is the nuclear charge of every member of a given iso-spectrumlevel series. Column 2 lists the experimental data taken from the NIST data base [31]. The NIST collected accepted data were obtained by different authors all around the world and critically evaluated the reliability of those
TABLE VI

Energy levels $\left[\mathrm{cm}^{-1}\right]$ for the $[\mathrm{He}] 2 s 3 s{ }^{3} S_{1}$ series of Be-like sequences.

\begin{tabular}{c|c|c|c}
\hline \hline$Z$ & $\begin{array}{c}\text { Experimental } \\
\text { results [31] }\end{array}$ & $\begin{array}{c}\text { Present } \\
\text { results }\end{array}$ & $\begin{array}{c}\text { MBPT } \\
\text { method [33] }\end{array}$ \\
\hline 4 & 52080.9 & 52086 & \\
5 & 129774 & 129765 & \\
6 & 238213 & 238221 & 238878 \\
7 & 377285 & 377274 & 377662 \\
8 & 546973 & 546946 & 547283 \\
9 & 747284 & 747290 & 747571 \\
10 & 978300 & 978355 & 978538 \\
11 & 1239974 & 1240330 & 1240225 \\
12 & 1532450 & 1531590 & 1532687 \\
13 & 1855760 & 1856250 & 1855990 \\
14 & 2210700 & 2210810 & 2210204 \\
15 & 2595600 & 2595510 & 2595410 \\
16 & 3011500 & 3011470 & 3011652 \\
17 & 3458700 & 3458540 & 3459100 \\
18 & & 3936770 & 3937848 \\
19 & & 4448800 & 4447996 \\
20 & & 4987890 & 4989632 \\
21 & & 5551910 & 5562939 \\
22 & 6160800 & 6160810 & 6167992 \\
23 & & 6794530 & 6804958 \\
24 & 7463000 & 7463000 & 7473998 \\
& & &
\end{tabular}

TABLE VII

Energy levels $\left[\mathrm{cm}^{-1}\right]$ for the $[\mathrm{Ne}] 3 s^{2} 3 d$ ${ }^{2} D_{3 / 2}$ series of Al-like sequences.

\begin{tabular}{c|c|c|c}
\hline \hline$Z$ & $\begin{array}{c}\text { Experimental } \\
\text { results [31] }\end{array}$ & $\begin{array}{c}\text { Present } \\
\text { results }\end{array}$ & $\begin{array}{c}\text { MBPT } \\
\text { method [9] }\end{array}$ \\
\hline 13 & 32435.5 & 32402.5 & \\
14 & 79338.5 & 79278.3 & \\
15 & 116875 & 117487 & \\
16 & 152133 & 151047 & \\
17 & 185863 & 186753 & \\
18 & 218593 & 217892 & 217980 \\
19 & 250663 & 250834 & \\
20 & 282356 & 283097 & 283270 \\
21 & 313860 & 314016 & \\
22 & 345315 & 343574 & 344199 \\
23 & 376897 & 378044 & \\
24 & 408640 & 408743 & 407623 \\
25 & 440725 & 440180 & \\
26 & 473223 & 473116 & 472279 \\
27 & 506230 & 507436 & \\
28 & 539839 & 539313 & 538960 \\
29 & 574180 & 574190 & \\
& & &
\end{tabular}


TABLE VIII

Energy levels $\left[\mathrm{cm}^{-1}\right]$ for the $[\mathrm{Ne}] 3 s^{2} 3 d$ ${ }^{2} D_{5 / 2}$ series of Al-like sequences.

\begin{tabular}{c|c|c|c}
\hline \hline$Z$ & $\begin{array}{c}\text { Experimental } \\
\text { results [31] }\end{array}$ & $\begin{array}{c}\text { Present } \\
\text { results }\end{array}$ & $\begin{array}{c}\text { MBPT } \\
\text { method [9] }\end{array}$ \\
\hline 13 & 32436.8 & 32443.4 & \\
14 & 79355 & 79171.4 & \\
15 & 116886 & 117548 & \\
16 & 152147 & 151180 & \\
17 & 185891 & 186808 & \\
18 & 218653 & 217838 & 218030 \\
19 & 250781 & 250792 & \\
20 & 282577 & 283282 & 283288 \\
21 & 314214 & 314627 & \\
22 & 345859 & 344682 & 344726 \\
23 & 377650 & 379521 & \\
24 & 409741 & 410272 & 408710 \\
25 & 442220 & 441401 & \\
26 & 475202 & 473948 & 408710 \\
27 & 508793 & 508611 & \\
28 & 543107 & 543184 & 542192 \\
29 & 578243 & 585846 & \\
& & &
\end{tabular}

TABLE IX Energy levels $\left[\mathrm{cm}^{-1}\right]$ for the $[\mathrm{Ne}] 4 p^{2} P_{1 / 2}$ series; [Ne] $5 d^{2} D_{5 / 2}$ series; and $[\mathrm{Ne}] 6 f^{2} F_{7 / 2}$ series of Na-like sequences.

\begin{tabular}{c|c|c|c|c|c|c}
\hline \hline$Z$ & \multicolumn{2}{|c|}{$[\mathrm{Ne}] 4 p^{2} P_{1 / 2}$} & \multicolumn{2}{c|}{$[\mathrm{Ne}] 5 d^{2} D_{5 / 2}$} & \multicolumn{2}{|c}{$[\mathrm{Ne}] 6 f^{2} F_{7 / 2}$} \\
\cline { 2 - 7 } & $T_{\text {exp }}[31]$ & $T_{\text {cal }}$ & $T_{\text {exp }}[31]$ & $T_{\text {cal }}$ & $T_{\text {exp }}[31]$ & $T_{\text {cal }}$ \\
\hline 11 & 30267 & 30277.8 & 37036.8 & 37033.9 & 38399.8 & 38399.1 \\
12 & 80619.5 & 80580.9 & 103420 & 103431 & 109062 & 109064 \\
13 & 143633 & 143666 & 188878 & 188866 & 201971 & 201970 \\
14 & 218267 & 218289 & 291498 & 291495 & 315230 & 315228 \\
15 & 304161 & 304140 & 410639 & 410649 & 448090 & 448090 \\
16 & 401169 & 401134 & 546059 & 546058 & 600189 & 600194 \\
17 & 509197 & 509228 & 697619 & 697616 & 771346 & 771343 \\
18 & 628219 & 628386 & 865252 & 865294 & 961470 & 961431 \\
19 & 758262 & 758597 & 1049130 & 1049130 & 1170440 & 1170430 \\
20 & 899290 & 899775 & 1249030 & 1249080 & 1398440 & 1398280 \\
21 & 1051640 & 1051640 & 1465130 & 1464910 & 1644980 & 1644730 \\
22 & 1214390 & 1215030 & 1697480 & 1697480 & 1910650 & 1910650 \\
23 & 1388410 & 1389190 & 1946500 & 1946010 & 2195100 & 2195300 \\
24 & 1573840 & 1574350 & 2211080 & 2210730 & 2499260 & 2498930 \\
25 & 1770400 & 1770440 & 2491700 & 2491540 & 2821800 & 2821490 \\
26 & 1977650 & 1977630 & 2788610 & 2788610 & 3163190 & 3163140 \\
27 & 2196500 & 2196500 & 3103000 & 3102610 & 3524500 & 3524500 \\
28 & 2426100 & 2426760 & 3434600 & 3433420 & 3906100 & 3905340 \\
29 & 2667490 & 2667620 & 3780600 & 3780600 & 4305000 & 4305000 \\
30 & 2920300 & 2920300 & 4145000 & 4145940 & 4724800 & 4724920 \\
31 & 3184600 & 3183920 & 4526100 & 4529430 & 5163500 & 5164580
\end{tabular}

data. Therefore, we took the data taken from the NIST data base for comparison. Column 3 lists the results obtained using WBEPM theory. The results obtained by Safronova et al. are contained in column 4. Using the RMBPT, Ref. [32] reported the energy levels of $n=2$ states of berylium-like ions with nuclear charges ranging from $Z=4-100$ [32]. In their calculations, both the Coulomb interaction and the Breit-Coulomb interaction are carried out to the second order. In the next year, the same group extended the theory to study the $2 l 3 l^{\prime}$ states of berylium-like ions. 16 even-parity $(2 s 3 s, 2 p 3 p, 2 s 3 d)$ excited states and 20 odd-parity $(2 s 3 p, 2 p 3 s, 2 p 3 d)$ excited states were studied in their work [33]. In general, their results are in good agreement with experimental data. Most deviations are smaller than $1000 \mathrm{~cm}^{-1}$, when several of them are $1000-4000 \mathrm{~cm}^{-1}$ and only a few are more than $4000 \mathrm{~cm}^{-1}$. The comparison shows that our results are at the same level with Safronova's ones. Table II gives the parameters for the two Al-like series and three Na-like series. The energy levels of these five series are listed in Tables VII-IX. For two Al-like series, the experimental data from $Z=13$ to $Z=22$ are used to obtain the parameters $n^{\prime}, \sigma$, and $g$. When we obtained the parameters $a_{i}(i=0-6)$ from the least-squares fitting, $Z=23,26,29$ are added for $[\mathrm{Ne}] 3 s^{2} 3 d^{2} D_{3 / 2}$ series and $Z=24,27,28$ are added for [Ne] $3 s^{2} 3 d^{2} D_{5 / 2}$ series. The results taken from Ref. [9] are given for comparison. For three Na-like series, the experimental data from $Z=11$ to $Z=17$ are used to obtain the parameters $n^{\prime}, \sigma$, and $g$. When obtained the parameters $a_{i}(i=0-6)$ from the least-squares fitting, $Z=21,27,30$ are added for $[\mathrm{Ne}] 4 p^{2} P_{1 / 2}$ series; $Z=22,26,29$ are added for [Ne] $5 d^{2} D_{5 / 2}$ series; and $Z=22,27,29$ are added for [Ne] $6 f$ ${ }^{2} F_{7 / 2}$ series.

The main source of error in the present method is the incomplete treatment of relativistic effects. We find that the deviations between nonrelativistic energies and the experimental data show an increasing trend with the increase in $Z$ along the iso-spectrum-level series. That is because the relativistic effects on the radial wave functions become more evident for high $Z$. Although the relative deviations caused by relativistic effects are very small, the absolute deviations will be little large ones. In present work, in order to improve the accuracy of our results, the relativistic corrections of an iso-spectrum-level series are included by a six-order polynomial in $Z$. By including the relativistic effects, deviations between our results and the experimental data become much smaller. However, this treatment is successful at present calculation. But for ions with much higher nuclear charge $Z$, the more precise consideration of relativistic effects is necessary.

In conclusion, employing the WBEPM theory, we calculated the energies for $\mathrm{Be}-$, Al-, and Na-like sequence. Equation (11) is derived to calculate the non-relativistic energies, and the relativistic corrections are taken into account by a six-order polynomial in nuclear charge $Z$. 
The present results are compared with available experimental data, and good agreements are obtained from the comparisons.

\section{References}

[1] A. Irimia, C.F. Fischer, J. Phys. B 37, 1659 (2004).

[2] C.F. Fischer, G. Tachiev, At. Data Nucl. Data Tables 87, 1 (2004).

[3] C.F. Fischer, G. Tachiev, A. Irimia, At. Data Nucl. Data Tables 92, 607 (2006).

[4] V.A. Dzuba, U.I. Safronova, W.R. Johnson, Phys. Rev. A 68, 032503 (2003).

[5] V.A. Dzuba, Phys. Rev. A 71, 062501 (2005).

[6] V.A. Dzuba, J.S.M. Ginges, Phys. Rev. A 73, 032503 (2006).

[7] V.A. Dzuba, V.V. Flambaum, J. Phys. B 40, 227 (2007).

[8] U.I. Safronova, W.R. Johnson, M.S. Safronova, J.R. Albritton, Phys. Rev. A 66, 022507 (2002).

[9] U.I. Safronova, C. Namba, J.R. Albritton, W.R Johnson, M.S. Safronova, Phys. Rev. A 65, 022507 (2002).

[10] U.I. Safronova, T.E. Cowan, M.S. Safronova, Phys. Lett. A 348, 293 (2006).

[11] U.I. Safronova, I.M. Savukov, M.S. Safronova, W.R. Johnson, Phys. Rev. A 68, 62505 (2003).

[12] H.C. Ho, W.R. Johnson, S.A. Blundell, M.S. Safronova, Phys. Rev. A 74, 022510 (2006).

[13] U.I. Safronova, W.R. Johnson, M.S. Safronova, Phys. Rev. A 76, 042504 (2007).

[14] S.A. Blundell, W.R. Johnson, M.S. Safronova, U.I. Safronova, Phys. Rev. A 77, 032507 (2008).

[15] N.W. Zheng, A New Outline of Atomic Theory, Jiang Su Education Press, Nan Jing, China 1988.

[16] N.W. Zheng, T. Wang, D.X. Ma, T. Zhou, J. Fan, Int. J. Quantum Chem. 98, 281 (2004).
[17] T.Y. Zhang, N.W. Zheng, D.X. Ma, Phys. Scr. 75, 763 (2007).

[18] D.X. Ma, N.W. Zheng, J. Fan, J. Phys. Chem. Ref. Data 33, 1013 (2004).

[19] D.X. Ma, N.W. Zheng, X. Lin, Spectrochim. Acta B 58, 1625 (2003).

[20] N.W. Zheng, T. Zhou, R.Y. Yang, T. Wang, D.X. Ma, Chem. Phys. 258, 37 (2000).

[21] N.W. Zheng, D.X. Ma, R.Y. Yang, T. Zhou, T.Wang, S. Han, J. Chem. Phys. 113, 1681 (2000).

[22] N.W. Zheng, Y.J. Sun, Sci. Chin. B 43, 113 (2000).

[23] N.W. Zheng, J. Fan, D.X. Ma, T. Wang, J. Phys. Soc. Jap. 72, 3091 (2003).

[24] N.W. Zheng, T. Wang, D.X. Ma, T. Zhou, Int. J. Quantum Chem. 87, 293 (2002).

[25] N.W. Zheng, Weakest Bound Electron Theory, University of Science and Technology of China press, to be published.

[26] N.W. Zheng, T. Wang, Int. J. Quantum Chem. 98, 495 (2004).

[27] N.W. Zheng, T. Wang, Chem. Phys. Lett. 376, 557 (2003).

[28] N.W. Zheng, T. Wang, Int. J. Quantum Chem. 93, 344 (2003).

[29] N.W. Zheng, T. Zhou, T. Wang, R.Y. Yang, Y.J. Sun, F. Wang, C.G. Chen, Phys. Rev. A 65, 052510 (2002).

[30] E.R. Davidson, S.A. Hagstrom, S.J. Chakrorty, V.M. Umar, C.F. Fischer, Phys. Rev. A 44, 7071 (1991).

[31] J.R. Fuhr, W.C. Martin, A. Musgrove, J. Sugar, W.L. Wiese, NIST Atomic Spectroscopic Database, http://physics.nist.gov [Physical Reference Data].

[32] M.S. Safronova, W.R. Johnson, U.I. Safronova, Phys. Rev. A 53, 4036 (1996).

[33] M.S. Safronova, W.R. Johnson, U.I. Safronova, J. Phys. B 30, 2375 (1997). 